Article

\title{
On the Non-Hypercyclicity of Normal Operators, Their Exponentials, and Symmetric Operators
}

\author{
Marat V. Markin and Edward S. Sichel * \\ Department of Mathematics, California State University, Fresno, 5245 N. Backer Avenue, M/S PB 108, \\ Fresno, CA 93740-8001, USA; mmarkin@csufresno.edu \\ * Correspondence: edsichel@mail.fresnostate.edu
}

Received: 23 August 2019; Accepted: 25 September 2019; Published: 27 September 2019

\begin{abstract}
We give a simple, straightforward proof of the non-hypercyclicity of an arbitrary (bounded or not) normal operator $A$ in a complex Hilbert space as well as of the collection $\left\{e^{t A}\right\}_{t>0}$ of its exponentials, which, under a certain condition on the spectrum of $A$, coincides with the $C_{0}$-semigroup generated by it. We also establish non-hypercyclicity for symmetric operators.
\end{abstract}

Keywords: hypercyclicity; scalar type spectral operator; normal operator; $C_{0}$-semigroup

MSC: Primary 47A16; 47B15; Secondary 47D06; 47D60; 34G10

\section{Introduction}

In [1], furnished is a straightforward proof of the non-hypercyclicity of an arbitrary (bounded or not) scalar type spectral operator $A$ in a complex Banach space as well as of the collection $\left\{e^{t A}\right\}_{t \geq 0}$ of its exponentials (see, e.g., [2]), the important particular case of a normal operator $A$ in a complex Hilbert space (see, e.g., [3,4]) following immediately.

Without the need to resort to the machinery of dual space, we provide a shorter, simpler, and more transparent direct proof for the normal operator case, in particular, generalizing the known result [5] [Corollary 5.31] for bounded normal operators, and further establish non-hypercyclicity for symmetric operators (see, e.g., [6]).

Definition 1 (Hypercyclicity). Let

$$
A: X \supseteq D(A) \rightarrow X
$$

$(D(\cdot)$ is the domain of an operator) be a (bounded or unbounded) linear operator in a (real or complex) Banach $\operatorname{space}(X,\|\cdot\|)$. A vector

$$
f \in C^{\infty}(A):=\bigcap_{n=0}^{\infty} D\left(A^{n}\right)
$$

$\left(A^{0}:=I, I\right.$ is the identity operator on $\left.X\right)$ is called hypercyclic if its orbit

$$
\operatorname{orb}(f, A):=\left\{A^{n} f\right\}_{n \in \mathbb{Z}_{+}}
$$

under $A\left(\mathbb{Z}_{+}:=\{0,1,2, \ldots\}\right.$ is the set of nonnegative integers) is dense in $X$.

Linear operators possessing hypercyclic vectors are said to be hypercyclic.

More generally, a collection $\{T(t)\}_{t \in J}$ ( $J$ is a nonempty indexing set) of linear operators in $X$ is called hypercyclic if it possesses hypercyclic vectors, i.e., such vectors $f \in \bigcap_{t \in J} D(T(t))$, whose orbit

$$
\{T(t) f\}_{t \in J}
$$


is dense in $X$.

$$
\text { Cf. }[5,7-12] \text {. }
$$

\section{Remark 1.}

- Clearly, hypercyclicity for a linear operator can only be discussed in a separable Banach space setting. Generally, for a collection of operators, this need not be the case.

- For a hypercyclic linear operator $A$, dense in $(X,\|\cdot\|)$ is the subspace $C^{\infty}(A)(c f .$, e.g., [1]), which, in particular, implies that any hypercyclic linear operator is densely defined (i.e., $\overline{D(A)}=X$ ).

- Bounded normal operators on a complex Hilbert space are known to be non-hypercyclic [5] [Corollary 5.31].

\section{Preliminaries}

Here, we briefly outline certain preliminaries essential for the subsequent discourse (for more, see, e.g., [13-15]).

Henceforth, unless specified otherwise, $A$ is a normal operator in a complex Hilbert space $(X,(\cdot, \cdot),\|\cdot\|)$ with strongly $\sigma$-additive spectral measure (the resolution of the identity) $E_{A}(\cdot)$ assigning to Borel sets of the complex plane $\mathbb{C}$ orthogonal projection operators on $X$ and having the operator's spectrum $\sigma(A)$ as its support [3,4].

Associated with a normal operator $A$ is the Borel operational calculus assigning to any Borel measurable function $F: \sigma(A) \rightarrow \mathbb{C}$ a normal operator

$$
F(A):=\int_{\sigma(A)} F(\lambda) d E_{A}(\lambda)
$$

with

$$
f \in D(F(A)) \Leftrightarrow \int_{\sigma(A)}|F(\lambda)|^{2} d\left(E_{A}(\lambda) f, f\right)<\infty,
$$

where $\left(E_{A}(\cdot) f, f\right)$ is a Borel measure, in which case $[3,4]$

$$
\|F(A) f\|^{2}=\int_{\sigma(A)}|F(\lambda)|^{2} d\left(E_{A}(\lambda) f, f\right)
$$

In particular,

$$
A^{n}=\int_{\sigma(A)} \lambda^{n} d E_{A}(\lambda), n \in \mathbb{Z}_{+}, \quad \text { and } \quad e^{t A}:=\int_{\sigma(A)} e^{t \lambda} d E_{A}(\lambda), t \in \mathbb{R} .
$$

Provided

$$
\sigma(A) \subseteq\{\lambda \in \mathbb{C} \mid \operatorname{Re} \lambda \leq \omega\}
$$

with some $\omega \in \mathbb{R}$, the collection of exponentials $\left\{e^{t A}\right\}_{t>0}$ is the $C_{0}$-semigroup generated by $A[4,16]$.

\section{Remark 2.}

- By [13] [Theorem 1], the orbits

$$
y(t)=e^{t A} f, t \geq 0, f \in \bigcap_{t \geq 0} D\left(e^{t A}\right)
$$

describe all weak/mild solutions of the abstract evolution equation

$$
y^{\prime}(t)=A y(t), t \geq 0,
$$

(see [17], cf. also [16] [Ch. II, Definition 6.3]). 
- The subspaces

$$
C^{\infty}(A) \text { and } \bigcap_{t \geq 0} D\left(e^{t A}\right)
$$

of all possible initial values for the corresponding orbits are dense in $X$ since they contain the subspace

$$
\bigcup_{\alpha>0} E_{A}\left(\Delta_{\alpha}\right) X, \text { where } \Delta_{\alpha}:=\{\lambda \in \mathbb{C}|| \lambda \mid \leq \alpha\}, \alpha>0,
$$

which is dense in $X$ and coincides with the class $\mathscr{E}^{\{0\}}(A)$ of the entire vectors of $A$ of exponential type (see, e.g., [18,19], cf. also [20]).

\section{Normal Operators and Their Exponentials}

We are to prove [1] [Corollary 4.1] directly generalizing in part [5] [Corollary 5.31].

Theorem 1 ([1] (Corollary 4.1)). An arbitrary normal, in particular self-adjoint, operator $A$ in a nonzero complex Hilbert space $(X,(\cdot, \cdot),\|\cdot\|)$ with spectral measure $E_{A}(\cdot)$ is not hypercyclic and neither is the collection $\left\{e^{t A}\right\}_{t \geq 0}$ of its exponentials, which, provided the spectrum of $A$ is located in a left half-plane

$$
\{\lambda \in \mathbb{C} \mid \operatorname{Re} \lambda \leq \omega\},
$$

with some $\omega \in \mathbb{R}$, is the $C_{0}$-semigroup generated by $A$.

Proof. Let $f \in C^{\infty}(A) \backslash\{0\}$ be arbitrary.

There are two possibilities: either

$$
E_{A}(\{\lambda \in \sigma(A)|| \lambda \mid>1\}) f \neq 0
$$

or

$$
E_{A}(\{\lambda \in \sigma(A)|| \lambda \mid>1\}) f=0 .
$$

In the first case, for any $n \in \mathbb{Z}_{+}$,

$$
\begin{aligned}
& \left\|A^{n} f\right\|^{2} \\
& =\int_{\sigma(A)}|\lambda|^{2 n} d\left(E_{A}(\lambda) f, f\right) \geq \int_{\{\lambda \in \sigma(A)|| \lambda \mid>1\}}|\lambda|^{2 n} d\left(E_{A}(\lambda) f, f\right) \\
& \geq \int_{\{\lambda \in \sigma(A)|| \lambda \mid>1\}} 1 d\left(E_{A}(\lambda) f, f\right)=\left(E_{A}(\{\lambda \in \sigma(A)|| \lambda \mid>1\}) f, f\right) \\
& =\left\|E_{A}(\{\lambda \in \sigma(A)|| \lambda \mid>1\}) f\right\|^{2}>0,
\end{aligned}
$$

which implies that the orbit $\operatorname{orb}(f, A)$ of $f$ under $A$ cannot approximate the zero vector, and hence, is not dense in $X$.

In the second case, since

$$
f=E_{A}(\{\lambda \in \sigma(A)|| \lambda \mid>1\}) f+E_{A}(\{\lambda \in \sigma(A)|| \lambda \mid \leq 1\}) f,
$$

we infer that

$$
f=E_{A}(\{\lambda \in \sigma(A)|| \lambda \mid \leq 1\}) f \neq 0
$$


and hence, for any $n \in \mathbb{Z}_{+}$,

$$
\begin{aligned}
& \left\|A^{n} f\right\|^{2}=\left\|A^{n} E_{A}(\{\lambda \in \sigma(A)|| \lambda \mid \leq 1\}) f\right\|^{2} \\
& =\int_{\{\lambda \in \sigma(A)|| \lambda \mid \leq 1\}}|\lambda|^{2 n} d\left(E_{A}(\lambda) f, f\right) \leq \int_{\{\lambda \in \sigma(A)|| \lambda \mid \leq 1\}} 1 d\left(E_{A}(\lambda) f, f\right) \\
& =\left(E_{A}(\{\lambda \in \sigma(A)|| \lambda \mid \leq 1\}) f, f\right)=\left\|E_{A}(\{\lambda \in \sigma(A)|| \lambda \mid \leq 1\}) f\right\|^{2}=\|f\|^{2},
\end{aligned}
$$

which also implies that the orbit $\operatorname{orb}(f, A)$ of $f$ under $A$, being bounded, is not dense in $X$ and completes the proof for the operator case.

Now, let us consider the case of the exponential collection $\left\{e^{t A}\right\}_{t \geq 0}$ assuming that $f \in \bigcap_{t \geq 0} D\left(e^{t A}\right) \backslash\{0\}$ is arbitrary.

There are two possibilities: either

$$
E_{A}(\{\lambda \in \sigma(A) \mid \operatorname{Re} \lambda>0\}) f \neq 0
$$

or

$$
E_{A}(\{\lambda \in \sigma(A) \mid \operatorname{Re} \lambda>0\}) f=0 .
$$

In the first case, for any $t \geq 0$,

$$
\begin{aligned}
& \left\|e^{t A} f\right\|^{2} \\
& =\int_{\sigma(A)}\left|e^{t \lambda}\right|^{2} d\left(E_{A}(\lambda) f, f\right)=\int_{\sigma(A)} e^{2 t \operatorname{Re} \lambda} d\left(E_{A}(\lambda) f, f\right) \\
& \geq \int_{\{\lambda \in \sigma(A) \mid \operatorname{Re} \lambda>0\}} e^{2 t \operatorname{Re} \lambda} d\left(E_{A}(\lambda) f, f\right) \geq \int_{\{\lambda \in \sigma(A) \mid \operatorname{Re} \lambda>0\}} 1 d\left(E_{A}(\lambda) f, f\right) \\
& =\left(E_{A}(\{\lambda \in \sigma(A) \mid \operatorname{Re} \lambda>0\}) f, f\right)=\left\|E_{A}(\{\lambda \in \sigma(A) \mid \operatorname{Re} \lambda>0\}) f\right\|^{2}>0,
\end{aligned}
$$

which implies that the orbit $\left\{e^{t A} f\right\}_{t \geq 0}$ of $f$ cannot approximate the zero vector, and hence, is not dense in $X$.

In the second case, since

$$
f=E_{A}(\{\lambda \in \sigma(A) \mid \operatorname{Re} \lambda>0\}) f+E_{A}(\{\lambda \in \sigma(A) \mid \operatorname{Re} \lambda \leq 0\}) f,
$$

we infer that

$$
f=E_{A}(\{\lambda \in \sigma(A) \mid \operatorname{Re} \lambda \leq 0\}) f \neq 0
$$

and hence, for any $t \geq 0$,

$$
\begin{aligned}
& \left\|e^{t A} f\right\|^{2}=\left\|e^{t A} E_{A}(\{\lambda \in \sigma(A) \mid \operatorname{Re} \lambda \leq 0\}) f\right\|^{2} \\
& =\int_{\{\lambda \in \sigma(A) \mid \operatorname{Re} \lambda \leq 0\}}\left|e^{t \lambda}\right|^{2} d\left(E_{A}(\lambda) f, f\right)=\int_{\{\lambda \in \sigma(A) \mid \operatorname{Re} \lambda \leq 0\}} e^{2 t \operatorname{Re} \lambda} d\left(E_{A}(\lambda) f, f\right) \\
& \leq \int_{\{\lambda \in \sigma(A) \mid \operatorname{Re} \lambda \leq 0\}} 1 d\left(E_{A}(\lambda) f, f\right)=\left(E_{A}(\{\lambda \in \sigma(A) \mid \operatorname{Re} \lambda \leq 0\}) f, f\right) \\
& =\left\|E_{A}(\{\lambda \in \sigma(A) \mid \operatorname{Re} \lambda \leq 0\}) f\right\|^{2}=\|f\|^{2},
\end{aligned}
$$


which also implies that the orbit $\left\{e^{t A} f\right\}_{t>0}$ of $f$, being bounded, is not dense on $X$ and completes the proof of the exponential case and the entire statement.

\section{Symmetric Operators}

The following generalizes in part [5] [Lemma 2.53 (a)] to the case of a densely defined unbounded linear operator in a Hilbert space.

Lemma 1. Let $A$ be a hypercyclic linear operator in a nonzero Hilbert space $(X,(\cdot, \cdot),\|\cdot\|)$ over the scalar field $\mathbb{F}$ of real or complex numbers (i.e., $\mathbb{F}=\mathbb{R}$ or $\mathbb{F}=\mathbb{C}$ ). Then

1. the adjoint operator $A^{*}$ has no eigenvalues, or equivalently, for any $\lambda \in \mathbb{F}$, the range of the operator $A-\lambda I$ is dense in $X$, i.e.,

$$
\overline{R(A-\lambda I)}=X
$$

$(R(\cdot)$ is the range of an operator);

2. provided the space $X$ is complex (i.e., $\mathbb{F}=\mathbb{C}$ ) and the operator $A$ is closed, the residual spectrum of $A$ is empty, i.e.,

$$
\sigma_{r}(A)=\varnothing
$$

\section{Proof.}

1. Let $f \in X$ be a hypercyclic vector for $A$.

We proceed by contradiction, assuming that the adjoint operator $A^{*}$, which exists since $A$ is densely defined (see Remark 1), has an eigenvalue $\lambda \in \mathbb{F}$, and hence,

$$
\exists g \in X \backslash\{0\}: A^{*} g=\lambda g
$$

which, in particular, implies that $g \in C^{\infty}\left(A^{*}\right):=\bigcap_{n=0}^{\infty} D\left(\left(A^{*}\right)^{n}\right)$ and

$$
\forall n \in \mathbb{N}:\left(A^{*}\right)^{n} g=\lambda^{n} g .
$$

In view of the above, we have inductively:

$$
\forall n \in \mathbb{N}:\left(A^{n} f, g\right)=\left(A^{n-1} f, A^{*} g\right)=\left(f,\left(A^{*}\right)^{n} g\right)=\left(f, \lambda^{n} g\right)=\bar{\lambda}^{n}(f, g),
$$

the conjugation being superfluous when the space is real.

Since $g \neq 0$, by the Riesz representation theorem (see, e.g., [21,22]), the hypercyclicity of $f$ implies that the set

$$
\left\{\left(A^{n} f, g\right)\right\}_{n \in \mathbb{N}}
$$

is dense in $\mathbb{F}$, which contradicts the fact that the same set

$$
\left\{\bar{\lambda}^{n}(f, g)\right\}_{n \in \mathbb{N}}
$$

is clearly not.

Thus, the adjoint operator $A^{*}$ has no eigenvalues.

The rest of the statement of part (1) immediately follows from the orthogonal sum decomposition

$$
X=\operatorname{ker}\left(A^{*}-\bar{\lambda} I\right) \oplus \overline{R(A-\lambda I)}, \lambda \in \mathbb{F},
$$

the conjugation being superfluous when the space is real, (see, e.g., [21]). 
2. Suppose that the space $X$ is complex (i.e., $\mathbb{F}=\mathbb{C}$ ) and the operator $A$ is closed. Recalling that

$$
\sigma_{r}(A)=\{\lambda \in \mathbb{C} \mid A-\lambda I \text { is one-to-one and } \overline{R(A-\lambda I)} \neq X\}
$$

(see, e.g., [21,23]), we infer from part (1) that

$$
\sigma_{r}(A)=\varnothing .
$$

We immediately arrive at the following

Proposition 1 (Non-Hypercyclicity Test). Any densely defined closed linear operator A in a nonzero complex Hilbert space $X$ with a nonempty residual spectrum (i.e., $\sigma_{r}(A) \neq \varnothing$ ) is not hypercyclic.

Now, we are ready to prove the subsequent.

Theorem 2. An arbitrary symmetric operator A in a complex Hilbert space $X$ is not hypercyclic.

Proof. Since

$$
A \subseteq A^{*},
$$

without loss of generality, we can regard the symmetric operator $A$ to be closed (see, e.g., [24]).

If both deficiency indices of the operator $A$ are equal to zero, $A$ is self-adjoint $\left(A=A^{*}\right)$ (see, e.g., [6]), and hence, by Theorem 1 , is not hypercyclic.

If at least one of the deficiency indices of the operator $A$ is nonzero, then

$$
\sigma_{r}(A) \neq \varnothing
$$

(see, e.g., [6,22]), and hence, by Proposition 1, $A$ is not hypercyclic.

\section{Some Examples}

\section{Example 1.}

1. In the complex Hilbert space $L_{2}(\mathbb{R})$, the self-adjoint differential operator $A:=i \frac{d}{d x}$ ( $i$ is the imaginary unit) with the domain

$$
D(A):=W_{2}^{1}(\mathbb{R}):=\left\{f \in L_{2}(\mathbb{R}) \mid f(\cdot) \in A C(\mathbb{R}), f^{\prime} \in L_{2}(\mathbb{R})\right\}
$$

$(A C(\cdot)$ is the set of absolutely continuous functions on an interval) is non-hypercyclic by Theorem 1 (cf. [1] [Corollary 5.1]).

2. In the complex Hilbert space $L_{2}(0, \infty)$, the symmetric differential operator $A:=i \frac{d}{d x}$ with the domain

$$
D(A):=\left\{f \in L_{2}(0, \infty) \mid f(\cdot) \in A C[0, \infty), f^{\prime} \in L_{2}(0, \infty), f(0)=0\right\}
$$

and deficiency indices $(0,1)$ is non-hypercyclic by Theorem 2 .

3. In the complex Hilbert space $L_{2}(0,2 \pi)$, the symmetric differential operator $A:=i \frac{d}{d x}$ with the domain

$$
D(A):=\left\{f \in L_{2}(0,2 \pi) \mid f(\cdot) \in A C[0,2 \pi], f^{\prime} \in L_{2}(0,2 \pi), f(0)=f(2 \pi)=0\right\}
$$

and deficiency indices $(1,1)$ is non-hypercyclic by Theorem 2 .

Cf. [6] (Sections 49 and 80). 
Author Contributions: Conceptualization, M.V.M.; methodology, M.V.M.; validation, E.S.S.; formal analysis, E.S.S.; investigation, M.V.M., E.S.S.; writing—original draft preparation, M.V.M., E.S.S.; writing-review and editing, M.V.M., E.S.S.; supervision, M.V.M.

Funding: This research received no external funding.

Conflicts of Interest: The authors declare no conflict of interest.

\section{References}

1. Markin, M.V. On the non-hypercyclicity of scalar type spectral operators and collections of their exponentials. arXiv 2018, arXiv:1807.07423.

2. Dunford, N.; Schwartz, J.T.; Bade, W.G.; Bartle, R.G. Linear Operators. Part III: Spectral Operators; Interscience Publishers: New York, NY, USA, 1971.

3. Dunford, N.; Schwartz, J.T.; Bade, W.G.; Bartle, R.G. Linear Operators. Part II: Spectral Theory. Self Adjoint Operators in Hilbert Space; Interscience Publishers: New York, NY, USA, 1963.

4. Plesner, A.I. Spectral Theory of Linear Operators; Nauka: Moscow, Russia, 1965. (In Russian)

5. Grosse-Erdmann, K.; Manguillot, A.P. Linear Chaos; Universitext; Springer: London, UK, 2011.

6. Akhiezer, N.I.; Glazman, I.M. Theory of Linear Operators in Hilbert Space; Dover Publications, Inc.: New York, NY, USA, 1993.

7. Bès, J.; Chan, K.C.; Seubert, S.M. Chaotic unbounded differentiation operators. Integral Equations Oper. Theory 2001, 40, 257-267. [CrossRef]

8. deLaubenfels, R.; Emamirad, H.; Grosse-Erdmann, K. Chaos for semigroups of unbounded operators. Math. Nachr. 2003, 261/262, 47-59. [CrossRef]

9. Guirao, A.J.; Montesinos, V.; Zizler, V. Open Problems in the Geometry and Analysis of Banach Spaces; Springer International Publishing: Cham, Switzerland, 2016.

10. Markin, M.V. On the chaoticity and spectral structure of Rolewicz-type unbounded operators. arXiv 2018, arXiv:1811.06640.

11. Markin, M.V. On general construct of chaotic unbounded linear operators in Banach spaces with Schauder bases. arXiv 2018, arXiv:1812.02294.

12. Rolewicz, S. On orbits of elements. Studia Math. 1969, 32, 17-22. [CrossRef]

13. Markin, M.V. On the strong smoothness of weak solutions of an abstract evolution equation. I. Differentiability. Appl. Anal. 1999, 73, 573-606 [CrossRef]

14. Markin, M.V. On the strong smoothness of weak solutions of an abstract evolution equation. II. Gevrey ultradifferentiability. IBID 2001, 78, 97-137 [CrossRef]

15. Markin, M.V. On the strong smoothness of weak solutions of an abstract evolution equation. III. Gevrey ultradifferentiability of orders less than one. IBID 2001, 78, 139-152. [CrossRef]

16. Engel, K.; Nagel, R. One-Parameter Semigroups for Linear Evolution Equations; Graduate Texts in Mathematics; Springer: New York, NY, USA, 2000; Volume 194.

17. Ball, J.M. Strongly continuous semigroups, weak solutions, and the variation of constants formula. Proc. Am. Math. Soc. 1977, 63, 370-373. [CrossRef]

18. Gorbachuk, V.I.; Knyazyuk, A.V. Boundary values of solutions of operator-differential equations. Russ. Math. Surv. 1989, 44, 67-111. [CrossRef]

19. Radyno, Y.V. The space of vectors of exponential type. Dokl. Akad. Nauk BSSR 1983, 27, 791-793.

20. Markin, M.V. On the Carleman ultradifferentiable vectors of a scalar type spectral operator. Methods Funct. Anal. Topol. 2015, 21, 361-369.

21. Markin, M.V. Elementary Operator Theory; De Gruyter Graduate; Walter de Gruyter GmbH: Berlin, Germany; Boston, MA, USA. (to appear).

22. Markin, M.V. Elementary Functional Analysis; De Gruyter Graduate; Walter de Gruyter GmbH: Berlin, Germany; Boston, MA, USA, 2018. 
23. Markin, M.V. On certain spectral features inherent to scalar type spectral operators. Methods Funct. Anal. Topol. 2017, 23, 60-65.

24. Dunford, N.; Schwartz, J.T.; Bade, W.G.; Bartle, R.G. Linear Operators. Part I: General Theory; Interscience Publishers: New York, NY, USA, 1958.

(C) 2019 by the authors. Licensee MDPI, Basel, Switzerland. This article is an open access article distributed under the terms and conditions of the Creative Commons Attribution (CC BY) license (http://creativecommons.org/licenses/by/4.0/). 